\title{
ENGINEERING GEOLOGY OF ALLUVIAL DEPOSITS IN YANGON AREA
}

\author{
Khin Soe Moe ${ }^{1}$ \\ ${ }^{1}$ Department of Engineering Geology, Yangon Technological University, Myanmar, \\ Tel: +95-9-773688781, e-mail: khinsoemoe.ytu@gmail.com
}

Received Date: November 7, 2017; Revised Date: March 25, 2019; Acceptance Date: March 27, 2019

\begin{abstract}
Two-thirds of the Yangon area is covered by alluvial deposits, which are weak in their strength and highly permeable. As Yangon area has undergone very rapid development and expansion, its expansion of new satellite towns is mostly built on the alluvial deposit. Engineering geology study of alluvial deposits in Yangon area is therefore needed to ascertain the nature and properties of alluvial deposits at different locations and at various depths. The distribution of alluvial deposits in Yangon starts from Kyaukmyinthar area to the Letkikkone and along the flood plain of Yangon river and Hlaing river. The alluvial deposits are categorized into valley fill, marine, and flat plain types. The most common deposit types are CL, CH, SM, SC, ML according to the Unified Soil Classification. Standard Penetration Test (SPT) is performed for this research and samples collected from boreholes are tested in the laboratory. Based on the SPT value and the laboratory results, the bearing capacity calculations are made for the foundation condition. Groundwater level is noted on each bore holes for the hydrological characteristics. The ultimate bearing capacity of valley fill deposits are more than $200 \mathrm{kPa}$ in Ahlon Township but the ultimate bearing capacity of most marine alluvium in Hlaingtharyar and N. Okkalarpa Townships are less than $100 \mathrm{kPa}$.
\end{abstract}

Keywords: Alluvial deposits, Bearing capacity, Engineering geology, Yangon area

\section{Introduction}

Alluvium is the general term for the detrital deposits made by rivers or streams or found on alluvial fans, flood plains, etc. It consists of a variety of materials, including fine particles of silt and clay and larger particles of sand and gravel and often contains organic matter that makes it a fertile soil. It does not include the subaqueous sediments of lakes and seas [1]. Otherwise all those deposits that are laid down by running water are called alluvial deposits. They are younger than the rock on which it lands and can cause confusion as to the age of the rock underneath and in the surrounding area [2]. Yangon is the largest trade city in Myanmar. Today Yangon has undergone very rapid development and expansion because of its socio-economic cum political significance. Therefore, it is required to expand the new satellite town. New satellite town are mostly built on the alluvial deposit. Moreover, the existing downtown areas are also built on alluvial deposits. Many existing structures in downtown are reconstructed as they are in dangerous condition. It is aware that close interrelation exists between the natural environment and area development. Therefore, engineering geology of alluvial deposits in Yangon area is needed to take up to ascertain the nature and properties of alluvial soil available at different locations of site at various depths. Alluvial deposits are mostly distributed in Yangon area. Two-third of the total area of Yangon is covered by alluvial deposits. Yangon is located in Lower Myanmar at the confluence of the Yangon and Bago rivers about $30 \mathrm{~km}$ away from the Gulf of Martaban .It is located between North Latitude $16^{\circ} 45^{\prime}$ and $17^{\circ} 00^{\prime}$ and East Longitude $96^{\circ} 00^{\prime}$ and $96^{\circ}$ 17 ', which refers to the map index 94 D/1. Its standard time zone is UTC/GMT $+6: 30$ hours. 


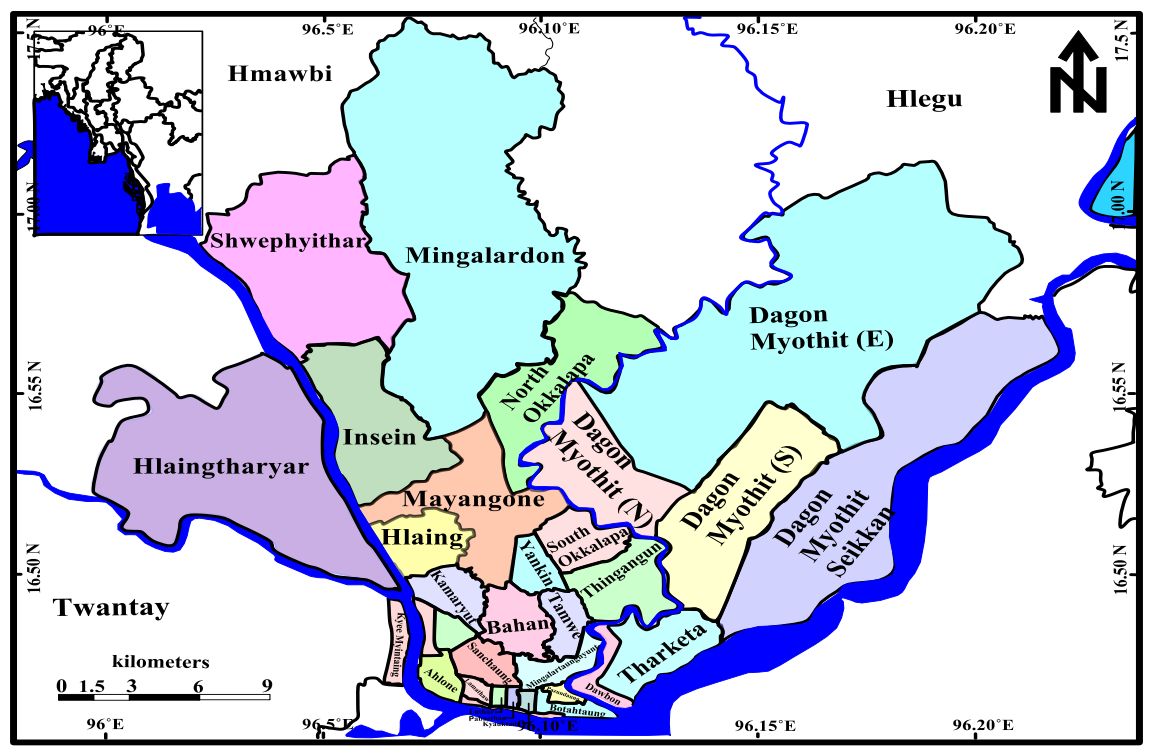

Figure 1. Location and township map of Yangon area [ 3 ]

There are thirty-four townships in Yangon area. Location and township map of Yangon is shown in Figure 1.

\section{Research Method}

For this research, Standard Penetration Test (SPT) is performed firstly and samples are collected from boreholes. The samples are tested in the laboratory for the physical and geotechnical properties of alluvial deposits in the Yangon area. Based on the SPT value and the laboratory results, the bearing capacity calculations are made for the foundation condition. Groundwater level is noted on each bore holes for the hydrological characteristics. This research will give many engineering characteristics of soils which are very valuable in determining the suitable foundation condition. It is also help in deciding regional planning and preparing the building code. Moreover, it can point out the groundwater potential, earthquake hazards and flood prone areas. Bore holes location map for study is shown in Figure 2.

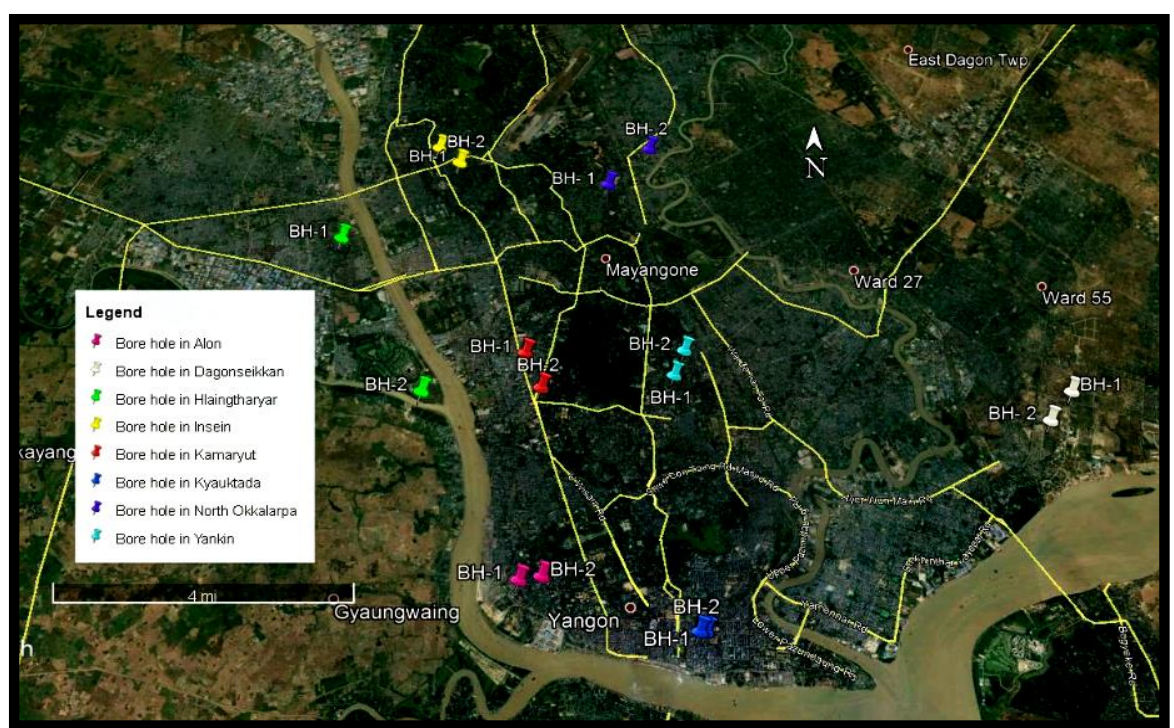

Figure 2. Bore holes location map of study area 


\section{Distribution of Alluvial Deposits in Yangon Area}

There are residual soil, alluvial (transported) soil and rock outcrop according to the topography and geological condition of Yangon area. Residual soil and rock outcrop mostly occurs along the Shwedagon- Mingalardon Ridge. They have good engineering properties and suitable for high - rise building. The alluvial deposits are weak in strength and high permeability because they are recent deposits. The alluvial deposits are widely distributed in Yangon area. The distribution of alluvial deposits starts from Kyaukmyinthar area to the Letkikkone and along the flood plain of Yangon river and Hlaing river. The lateral extent is about $130 \mathrm{~km}$ at Taikkyi section and about $210 \mathrm{~km}$ at the central Yangon section. The distribution of alluvial deposits in Yangon area is shown in Figure 3. The alluvial deposit in Yangon area is categorized into valley fill alluvial deposits, marine alluvial deposits, and flat plain alluvial deposits. Valley fill alluvial deposits are found in Insein, Hlaing Kamayut, Ahlon, Kyauktada and Botahtaung, Lanmadaw, Sanchaung Townships. Flat plain alluvial deposits are found in North Okkalapa, South Okkalapa, Dagon Myothit, Thingangyun, Thaketa, Dawbon Townships. Marine alluvial deposits are found in Simalaik, Bayintnaung, Shwepyithar, Hlaingtharyar, Hlagwa, Htatabin, Dala, Tharkata, and Dagon Seikkan Townships.

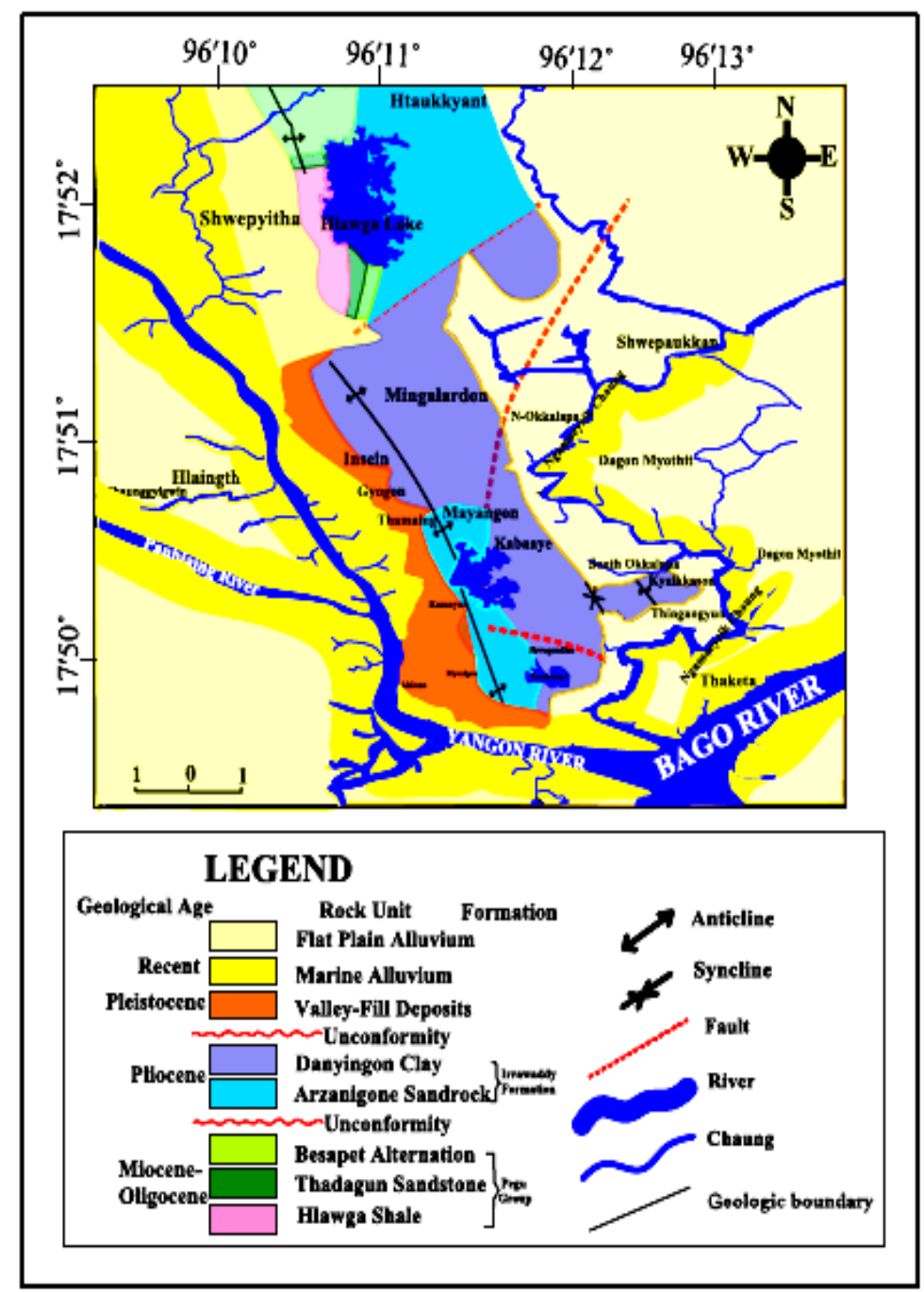

Figure 3. Distribution of alluvial deposits in Yangon area 


\section{Hydrological Characteristics of Alluvial Deposits in Yangon Area}

\section{Groundwater in Flat Plain Deposits}

Groundwater is found in the unconsolidated layer of alluvial deposits along the Ngamoeyeik creek and surrounding area. Length of alluvial deposits is about $29 \mathrm{~km}$ in north- south direction and about 3 to $10 \mathrm{~km}$ in east -west direction.

Alluvial deposits are mainly composed of fine to medium grained sand, blunish grey silt and clays. According to the well log data, the thickness is about 2 to $20 \mathrm{~m}$ varying from one place to another. Water from these deposits gives low yield and has been highly contaminated. Most of the wells are hand dug wells. Water table of this aquifer is found at $1.0 \mathrm{~m}$ to $7.5 \mathrm{~m}$ below the surface. At nearby area along the Ngamoeyeik creek, hand-dug wells are not usually dug due to poor quality of water.

\section{Groundwater in Valley Fill Deposits}

Generally, the best aquifers are observed in the valley fill deposits situated on the western flank of the Shwedagon ridge which serve as main aquifer of groundwater. The length of the valley fill deposits from north to south varies about 2 to $3.5 \mathrm{~km}$.

The valley fill deposits consist mainly of fine to coarse sands and gravel. Clays lens may interrupt these beds. These deposits have the vertical succession of coarse sand to gravel near the bottom to silt and clay at the top [4]. These deposits are principal aquifers in the western Yangon. The depth of the aquifer is generally noted as $12 \mathrm{~m}$ to $36 \mathrm{~m}$ in this area.

The valley filled deposits serve as good aquifer. In general, thickness of water bearing horizon ranges from $12 \mathrm{~m}$ to $31 \mathrm{~m}$. Water tapped from the shallow horizon of this aquifer (less than $40 \mathrm{~m}$ ) yield relatively high, being in the range of 120 to $410 \mathrm{gpm}$ for 8 inches diameter well in Botahtaung. In Lanmadaw Township, the water bearing horizon is encountered at $30 \mathrm{~m}$. In Ahlon, the groundwater yield from this horizon range $1000 \mathrm{gph}$ to $11000 \mathrm{gph}$. In Sanchaung, the depth of the water bearing horizon ranges from $21 \mathrm{~m}$ to $39 \mathrm{~m}$ with the thickness of $6 \mathrm{~m}$ to $18 \mathrm{~m}$ and water yields from this horizon is $6000 \mathrm{gph}$ to 10000 gph. In Hlaing, the depth of the water bearing horizon ranges from $12 \mathrm{~m}$ to $45 \mathrm{~m}$ and the thickness of the horizon is within the range of $6 \mathrm{~m}$ to $30 \mathrm{~m}$.

\section{Groundwater in Marine Alluvial Deposits}

Composition of marine alluvial deposits consists of marine clay, silty clay with high organic content from decaying vegetation. In Hlaingtharyar, water bearing horizon is found at the depth of $75 \mathrm{~m}$ with the thickness $12 \mathrm{~m}$ which yields $1000 \mathrm{gph}$. Also found shallow aquifer at the depth of $24 \mathrm{~m}$ to $42 \mathrm{~m}$ which yield $200 \mathrm{gph}$ to $3000 \mathrm{gph}$. In some places of Hlaingtharyar, salt water intrusion is found along the Hlaing and Panhlaing rivers.

\section{Geotechnical Characteristics of Alluvial Deposits in Yangon Area}

As alluvial deposits are widely distributed in Yangon area, they have different physical and geotechnical properties since the depositional background are different. To know the geotechnical properties, Standard Penetration Test (SPT) is performed firstly and samples are collected. The density test, moisture content, grain size distribution, hydrometer analysis, Atterberg limits,and direct shear tests are performed in the laboratory to evaluate the geotechnical properties of alluvial soil. Moreover, Standard Penetration Test N-value has to make correction since the Standard Penetration Test can have many uncertainties. 


\section{Geotechnical Characteristics of Valley Fill Deposits}

Valley fill deposits occupy the synclinal valley which is situated to the west of the Yangon ridge. These were probably deposited by the Proto Hlaing river in Pleistocene and sub-recent time as a channel deposit. At present, they have been partially uplifted along the margin of the anticline [4].They consist of a thick sequence of loose, highly pervious, interbedded sands and fine to very coarse gravels. SW, SM and CL soil types a mostly occurred in the valley fill alluvium. In the upper part of these deposits, the percentage of sand and silt are high. At the deeper portion, sand is mostly abundant sometimes with very little amount of gravel is observed. Tables 1, 2, 3, and 4 show the summary of geotechnical characteristics of valley fill deposits in Yangon area.

Table 1. Summary of Geotechnical Characteristics of Valley Fill Deposits in Kamaryut Township in Yangon Area

\begin{tabular}{|c|c|c|c|c|c|c|c|c|c|c|c|}
\hline \multirow[b]{2}{*}{ No. } & \multirow[b]{2}{*}{$\begin{array}{c}\text { Depth } \\
\text { (m) }\end{array}$} & \multirow{2}{*}{$\begin{array}{c}\text { Wet } \\
\text { Unit } \\
\text { Weight } \\
\text { kN/m } \mathbf{m}^{3}\end{array}$} & \multirow{2}{*}{$\begin{array}{c}\text { Dry } \\
\text { Unit } \\
\text { Weight } \\
\text { kN/m } \mathbf{m}^{3}\end{array}$} & \multirow{2}{*}{$\begin{array}{c}\text { SPT } \\
\mathbf{N} \\
\text { Value }\end{array}$} & \multirow[b]{2}{*}{ Lithology } & \multicolumn{3}{|c|}{$\begin{array}{c}\text { Atterberg } \\
\text { Limits }\end{array}$} & \multirow{2}{*}{$\begin{array}{c}\text { Moisture } \\
\text { Content } \\
(\%)\end{array}$} & \multicolumn{2}{|c|}{$\begin{array}{c}\text { Shear } \\
\text { Strength }\end{array}$} \\
\hline & & & & & & $\begin{array}{l}\text { L.L } \\
\%\end{array}$ & $\begin{array}{l}\text { P.L } \\
\%\end{array}$ & P.I & & $\begin{array}{c}\mathrm{c} \\
\mathrm{kPa}\end{array}$ & $\begin{array}{l}\phi \\
\left({ }^{\circ}\right)\end{array}$ \\
\hline \multirow{5}{*}{ BH-1 } & $0-2$ & 17.3 & 13.8 & $8-15$ & clayey silt & - & - & - & $23-27$ & - & - \\
\hline & $2-4$ & 18.2 & 14.6 & $16-18$ & clayey silt & 43 & 24 & 19 & $22-23$ & 1.2 & 15 \\
\hline & $4-6$ & 18.9 & 15.6 & $18-20$ & clayey silt & 46 & 25 & 21 & $19-22$ & 1.6 & 14 \\
\hline & $6-8$ & 19.3 & 16.3 & $20-21$ & clayey silt & 45 & 23 & 22 & $18-20$ & 2 & 14 \\
\hline & $8-10$ & 19.4 & 16.5 & 21 & sandy silt & 45 & 24 & 21 & 20 & 2 & 13 \\
\hline \multirow{5}{*}{ BH-2 } & $0-2$ & 17.4 & 14 & $10-15$ & silty sand & - & - & - & $23-26$ & - & - \\
\hline & $2-4$ & 18.2 & 15 & $15-18$ & silty sand & 39 & 23 & 16 & $20-23$ & 1.0 & 20 \\
\hline & $4-6$ & 19 & 16 & $19-20$ & $\begin{array}{l}\text { Silty } \\
\text { Sand }\end{array}$ & 45 & 25 & 20 & $18-20$ & 1.4 & 16 \\
\hline & $6-8$ & 19.2 & 16.3 & $20-21$ & clayey silt & 46 & 25 & 21 & $17-18$ & 1.0 & 23 \\
\hline & $8-10$ & 19.5 & 16.3 & 22 & clayey silt & 46 & 25 & 21 & 17 & 1.4 & 25 \\
\hline
\end{tabular}

Table 2. Summary of Geotechnical Characteristics of Valley Fill Deposits in Insein Township in Yangon Area

\begin{tabular}{|c|c|c|c|c|c|c|c|c|c|c|c|}
\hline \multirow[b]{2}{*}{ No. } & \multirow[b]{2}{*}{$\begin{array}{c}\text { Depth } \\
\text { (m) }\end{array}$} & \multirow{2}{*}{$\begin{array}{c}\text { Wet } \\
\text { Unit } \\
\text { Weight } \\
\text { kN/m } \mathbf{m}^{3}\end{array}$} & \multirow{2}{*}{$\begin{array}{c}\text { Dry } \\
\text { Unit } \\
\text { Weight } \\
\text { kN/m } \mathbf{m}^{3}\end{array}$} & \multirow{2}{*}{$\begin{array}{c}\text { SPT } \\
\mathbf{N} \\
\text { Value }\end{array}$} & \multirow[b]{2}{*}{ Lithology } & \multicolumn{3}{|c|}{$\begin{array}{c}\text { Atterberg } \\
\text { Limits }\end{array}$} & \multirow{2}{*}{$\begin{array}{c}\text { Moisture } \\
\text { Content } \\
(\%)\end{array}$} & \multicolumn{2}{|c|}{$\begin{array}{c}\text { Shear } \\
\text { Strength }\end{array}$} \\
\hline & & & & & & $\begin{array}{l}\text { L.L } \\
\%\end{array}$ & $\begin{array}{l}\text { P.L } \\
\%\end{array}$ & P.I & & $\begin{array}{c}\mathrm{c} \\
\mathrm{kPa}\end{array}$ & $\begin{array}{l}\phi \\
\left({ }^{\circ}\right)\end{array}$ \\
\hline \multirow{5}{*}{ BH-1 } & $0-2$ & 17.4 & 13.6 & $8-13$ & sandy silt & - & - & - & $23-24$ & - & - \\
\hline & $2-4$ & 17.6 & 14 & 16 & clayey silt & 43 & 24 & 19 & $21-23$ & 1.0 & 21 \\
\hline & $4-6$ & 17.8 & 14.5 & $12-14$ & clayey silt & 45 & 23 & 22 & $20-21$ & 1.0 & 18 \\
\hline & $6-8$ & 17 & 13.4 & 16 & clayey silt & 45 & 23 & 22 & $20-21$ & 1.4 & 15 \\
\hline & $8-10$ & 17.9 & 14.6 & 16 & clayey silt & 39 & 23 & 16 & 22 & 1.3 & 16 \\
\hline \multirow{5}{*}{ BH-2 } & $0-2$ & 17.1 & 13.7 & $11-15$ & sandy silt & - & - & - & $23-28$ & - & - \\
\hline & $2-4$ & 17.5 & 14.3 & $16-17$ & clayey silt & 44 & 24 & 20 & $23-24$ & 1 & 6 \\
\hline & $4-6$ & 18.1 & 13.7 & 19 & $\begin{array}{l}\text { clayey } \\
\text { silt }\end{array}$ & 43 & 22 & 21 & $25-27$ & 1 & 6 \\
\hline & $6-8$ & 19.3 & 15.7 & 18 & clayey silt & 42 & 22 & 20 & $23-27$ & 1.1 & 6 \\
\hline & $8-10$ & 18.6 & 15.3 & 17 & clayey silt & - & - & - & 25 & 1.3 & 10 \\
\hline
\end{tabular}


Table 3. Summary of Geotechnical Characteristics of Valley Fill Deposits in Ahlon Township in Yangon Area

\begin{tabular}{|c|c|c|c|c|c|c|c|c|c|c|c|}
\hline \multirow{2}{*}{ No. } & \multirow[b]{2}{*}{$\begin{array}{c}\text { Depth } \\
\text { (m) }\end{array}$} & \multirow{2}{*}{$\begin{array}{c}\text { Wet } \\
\text { Unit } \\
\text { Weight } \\
\text { kN/m } \mathbf{m}^{3}\end{array}$} & \multirow{2}{*}{$\begin{array}{c}\text { Dry } \\
\text { Unit } \\
\text { Weight } \\
\text { kN/m³ }\end{array}$} & \multirow{2}{*}{$\begin{array}{c}\text { SPT } \\
\mathbf{N} \\
\text { Value }\end{array}$} & \multirow{2}{*}{ Lithology } & \multicolumn{3}{|c|}{$\begin{array}{l}\text { Atterberg } \\
\text { Limits }\end{array}$} & \multirow{2}{*}{$\begin{array}{c}\text { Moisture } \\
\text { Content } \\
(\%)\end{array}$} & \multicolumn{2}{|c|}{$\begin{array}{l}\text { Shear } \\
\text { Strength }\end{array}$} \\
\hline & & & & & & $\begin{array}{c}\text { L.L } \\
\%\end{array}$ & $\begin{array}{l}\text { P.L } \\
\%\end{array}$ & P.I & & $\begin{array}{c}\mathrm{c} \\
\mathrm{kPa}\end{array}$ & $\begin{array}{l}\phi \\
\left({ }^{\circ}\right)\end{array}$ \\
\hline \multirow{5}{*}{ BH-1 } & $0-2$ & 16.86 & 13.26 & $4-5$ & silty sand & - & - & - & 28.3 & 10 & 8 \\
\hline & $2-4$ & 16.76 & 13.33 & $3-6$ & sandy and & 43 & 24 & 19 & $23-27$ & 13 & 13 \\
\hline & $4-6$ & 18.3 & 14.77 & $3-16$ & clayey silt & 42 & 23 & 19 & $22-24$ & 20 & 7 \\
\hline & $6-8$ & 18.72 & 15.49 & $17-18$ & clayey silt & 42 & 24 & 18 & $20-21$ & 29 & 8 \\
\hline & $8-10$ & 18.55 & 15.23 & 18 & clayey silt & 44 & 23 & 21 & $21-22$ & 30 & 8 \\
\hline \multirow{5}{*}{ BH-2 } & $0-2$ & 17.91 & 14.3 & $12-18$ & clayey silt & - & - & - & $22-27$ & 28 & 5 \\
\hline & $2-4$ & 18.5 & 15.2 & 18 & $\begin{array}{l}\text { clayey } \\
\text { and sandy } \\
\text { silt }\end{array}$ & 49 & 25 & 24 & $21-23$ & 25 & 6 \\
\hline & $4-6$ & 18.4 & 15 & 18 & $\begin{array}{l}\text { clayey } \\
\text { and sandy } \\
\text { silt }\end{array}$ & 47 & 24 & 23 & $20-23$ & 30 & 3 \\
\hline & $6-8$ & 19 & 15.9 & 19 & clayey silt & 49 & 25 & 24 & $19-21$ & 33 & 4 \\
\hline & $8-10$ & 19.2 & 16.1 & 20 & clayey silt & 51 & 26 & 25 & 19 & 33 & 4 \\
\hline
\end{tabular}

Table 4. Summary of Geotechnical Characteristics of Valley Fill Deposits in Kyauktada Township in Yangon Area

\begin{tabular}{|c|c|c|c|c|c|c|c|c|c|c|c|}
\hline \multirow{2}{*}{ No. } & \multirow[b]{2}{*}{$\begin{array}{c}\text { Dep } \\
\text { th } \\
(\mathbf{m})\end{array}$} & \multirow{2}{*}{$\begin{array}{c}\text { Wet } \\
\text { Unit } \\
\text { Weight } \\
\text { kN/m } \mathbf{m}^{3}\end{array}$} & \multirow{2}{*}{$\begin{array}{c}\text { Dry } \\
\text { Unit } \\
\text { Weight } \\
\text { kN/m } \mathbf{m}^{3}\end{array}$} & \multirow{2}{*}{$\begin{array}{c}\text { SPT } \\
\mathbf{N} \\
\text { Value }\end{array}$} & \multirow{2}{*}{ Lithology } & \multicolumn{3}{|c|}{$\begin{array}{l}\text { Atterberg } \\
\text { Limits }\end{array}$} & \multirow{2}{*}{$\begin{array}{c}\text { Moisture } \\
\text { Content } \\
(\%)\end{array}$} & \multicolumn{2}{|c|}{$\begin{array}{l}\text { Shear } \\
\text { Strength }\end{array}$} \\
\hline & & & & & & $\begin{array}{l}\text { L.L } \\
\%\end{array}$ & $\begin{array}{l}\text { P.L } \\
\%\end{array}$ & P.I & & $\begin{array}{c}\mathrm{c} \\
\mathrm{kPa}\end{array}$ & $\begin{array}{c}\phi \\
\left(^{\circ}\right)\end{array}$ \\
\hline \multirow{5}{*}{ BH-1 } & $0-2$ & 17.5 & 13.3 & $4-8$ & sandy silt & - & - & - & 25.6 & 4 & 28 \\
\hline & $2-4$ & 17.8 & 13.6 & $8-13$ & sandy silt & 41 & 24 & 17 & 23.8 & 6 & 29 \\
\hline & $4-6$ & 18.2 & 14.9 & 16 & clayey silt & 42 & 24 & 18 & 22.6 & 21 & 15 \\
\hline & $6-8$ & 19 & 15.7 & 19 & clayey silt & 42 & 24 & 18 & 20.3 & 24 & 8 \\
\hline & $8-10$ & 19.4 & 16.4 & 20 & clayey silt & 43 & 25 & 18 & 19 & 29 & 14 \\
\hline \multirow{5}{*}{ BH-2 } & $0-2$ & 17.4 & 13.5 & $6-10$ & clayey silt & - & - & - & 25.9 & 21 & 14 \\
\hline & $2-4$ & 17.8 & 14.5 & 16 & clayey silt & 41 & 24 & 17 & 23.4 & 6 & 31 \\
\hline & $4-6$ & 17.5 & 14 & 12 & clayey silt & - & - & - & 25 & 21 & 16 \\
\hline & 6-8 & 17.7 & 13.9 & 13 & sandy silt & 39 & 23 & 16 & 26.7 & 5 & 27 \\
\hline & $8-10$ & 17.8 & 14.4 & 14 & silty sand & - & - & - & 24.5 & 4 & 30 \\
\hline
\end{tabular}

\section{Geotechnical Characteristics of Marine Alluvial Deposits}

Marine alluvial deposits are also found in the coastal planes, the river valleys and the deltas of the plateau region. Most of marine alluvial deposits are marine clay, silty clay with high organic content from decaying vegetation [5]. Tables 5 and 6 show the summary of geotechnical characteristics of marine alluvial deposits in Yangon area. 
Table 5. Summary of Geotechnical Characteristics of Marine Alluvium in Dagon Seikkan Township in Yangon Area

\begin{tabular}{|c|c|c|c|c|c|c|c|c|c|c|c|}
\hline \multirow[b]{2}{*}{ No. } & \multirow[b]{2}{*}{$\begin{array}{c}\text { Dept } \\
\text { h } \\
(\mathbf{m})\end{array}$} & \multirow{2}{*}{$\begin{array}{c}\text { Wet Unit } \\
\text { Weight } \\
\text { kN/m }\end{array}$} & \multirow{2}{*}{$\begin{array}{c}\text { Dry } \\
\text { Unit } \\
\text { Weight } \\
\text { kN/m } \\
\end{array}$} & \multirow{2}{*}{$\begin{array}{c}\text { SPT } \\
\mathbf{N} \\
\text { Value }\end{array}$} & \multirow[b]{2}{*}{ Lithology } & \multicolumn{3}{|c|}{$\begin{array}{c}\text { Atterberg } \\
\text { Limits }\end{array}$} & \multirow{2}{*}{$\begin{array}{c}\text { Moisture } \\
\text { Content } \\
(\%)\end{array}$} & \multicolumn{2}{|c|}{$\begin{array}{c}\text { Shear } \\
\text { Strength }\end{array}$} \\
\hline & & & & & & $\underset{\%}{\mathbf{L . L}}$ & $\begin{array}{c}\text { P.L } \\
\%\end{array}$ & P.I & & $\begin{array}{c}\mathrm{c} \\
\mathrm{kPa}\end{array}$ & $\begin{array}{l}\phi \\
\left(^{\circ}\right)\end{array}$ \\
\hline \multirow{9}{*}{$\begin{array}{l}\mathrm{BH}- \\
1\end{array}$} & $0-2$ & 17.9 & 13 & 5 & lean clay & - & - & - & 37 & - & - \\
\hline & $2-4$ & 17.9 & 13 & 5 & lean clay & 39 & 24 & 15 & 37 & - & - \\
\hline & $4-6$ & 17.8 & 13 & 3 & lean clay & - & - & - & 37 & - & - \\
\hline & $6-8$ & 17.4 & 15.7 & 5 & lean clay & 43 & 24 & 19 & 42 & - & - \\
\hline & $8-10$ & 18 & 12.6 & 5 & sandy silt & - & - & - & 37 & - & - \\
\hline & $20-30$ & 19.2 & 16 & $16-25$ & silty sand & - & - & - & 24 & - & - \\
\hline & $30-40$ & 18.5 & 13.8 & $9-26$ & sandy clay & - & - & - & 29 & - & - \\
\hline & $40-50$ & 19.7 & 15.5 & $14-42$ & silty sand & 43 & 18 & 25 & 25 & - & - \\
\hline & $60-70$ & 21 & 14 & $16-50$ & fat clay & 43 & 25 & 18 & 23 & - & - \\
\hline \multirow{10}{*}{$\begin{array}{l}\mathrm{BH}- \\
2\end{array}$} & $0-2$ & 18.14 & 13.5 & 5 & clay & - & - & - & 34 & - & - \\
\hline & $2-4$ & 17 & 16.6 & 5 & clay & - & - & - & 46 & - & - \\
\hline & $4-6$ & 18 & 13 & 3 & lean clay & 43 & 22 & 21 & 40 & - & - \\
\hline & $6-8$ & 18.3 & 13.4 & 11 & lean clay & - & - & - & 34 & - & - \\
\hline & $8-10$ & 17.6 & 15 & 14 & lean clay & - & - & - & 36 & - & - \\
\hline & $10-15$ & 21 & 17.5 & $11-33$ & silty sand & - & - & - & 15 & - & - \\
\hline & $20-30$ & 18.5 & 14 & $19-37$ & silty sand & 37 & 24 & 13 & 25 & - & - \\
\hline & $30-40$ & 19.4 & 15 & $26-45$ & $\begin{array}{l}\text { clayey } \\
\text { sand }\end{array}$ & - & - & - & 29 & - & - \\
\hline & $40-45$ & 20.2 & 15.6 & $26-43$ & silty sand & 55 & 25 & 30 & 19.5 & - & - \\
\hline & $45-60$ & 18.8 & 16 & $19-45$ & fat clay & 63 & 32 & 31 & 36 & - & - \\
\hline
\end{tabular}

Table 6. Summary of Geotechnical Characteristics of Marine Alluvium in Hlaingtharyar Township in Yangon Area

\begin{tabular}{|c|c|c|c|c|c|c|c|c|c|c|c|}
\hline \multirow{2}{*}{ No. } & \multirow{2}{*}{$\begin{array}{c}\text { Depth } \\
\text { (m) }\end{array}$} & \multirow{2}{*}{$\begin{array}{c}\text { Wet Unit } \\
\text { Weight } \\
\text { kN/m³ }\end{array}$} & \multirow{2}{*}{$\begin{array}{c}\text { Dry } \\
\text { Unit } \\
\text { Weight } \\
\text { kN/m³ }\end{array}$} & \multirow{2}{*}{$\begin{array}{c}\text { SPT } \\
\mathbf{N} \\
\text { Value }\end{array}$} & \multirow{2}{*}{ Lithology } & \multicolumn{3}{|c|}{$\begin{array}{c}\text { Atterberg } \\
\text { Limits }\end{array}$} & \multirow{2}{*}{$\begin{array}{c}\text { Moisture } \\
\text { Content } \\
(\%)\end{array}$} & \multicolumn{2}{|c|}{$\begin{array}{c}\text { Shear } \\
\text { Strength }\end{array}$} \\
\hline & & & & & & $\begin{array}{c}\text { L.L } \\
\%\end{array}$ & $\begin{array}{c}\text { P.L } \\
\%\end{array}$ & P.I & & $\begin{array}{c}\mathrm{c} \\
\mathrm{kPa}\end{array}$ & $\begin{array}{c}\phi \\
\left(^{\circ}\right)\end{array}$ \\
\hline \multirow{10}{*}{$\begin{array}{l}\text { BH- } \\
1\end{array}$} & $0-2$ & 19 & 13 & 2 & lean clay & - & - & - & 37 & - & - \\
\hline & $2-4$ & 17.9 & 12.7 & 4 & lean clay & 74 & 37 & 37 & 37 & 12 & 2 \\
\hline & $4-6$ & 17.1 & 11.6 & 2 & fat clay & 68 & 31 & 37 & 37 & 10 & 1 \\
\hline & $6-8$ & 17.2 & 11.9 & 4 & fat clay & 43 & 24 & 19 & 42 & 6 & 1 \\
\hline & $8-10$ & 17.6 & 12.2 & 3 & fat clay & 70 & 31 & 39 & 37 & 7 & 1 \\
\hline & 10-13 & 17.3 & 12.2 & 9 & fat clay & 61 & 29 & 32 & 27 & 5 & 1 \\
\hline & $16-20$ & 16.8 & 11.9 & 13 & fat clay & 60 & 32 & 28 & 24 & 8 & 1 \\
\hline & $20-23$ & 17 & 11.5 & 7 & fat clay & 62 & 30 & 32 & 29 & 7 & 1 \\
\hline & $23-26$ & 19.4 & 14.9 & 12 & fat clay & 44 & 24 & 20 & 25 & 22 & 1 \\
\hline & $26-30$ & 19.36 & 15.8 & 42 & $\begin{array}{l}\text { Clayey } \\
\text { sand }\end{array}$ & - & - & - & 28 & 15 & 19 \\
\hline \multirow{11}{*}{$\begin{array}{l}\mathrm{BH}- \\
2\end{array}$} & $0-2$ & 18.1 & 13.35 & 1 & lean clay & - & - & - & 34 & - & - \\
\hline & $2-4$ & 18.1 & 13.35 & 3 & lean clay & 60 & 23 & 37 & 35 & 9 & 1 \\
\hline & $4-6$ & 17.5 & 12.5 & 4 & lean clay & 52 & 26 & 26 & 39 & 6 & 1 \\
\hline & $6-8$ & 17.2 & 12 & 3 & lean clay & 53 & 27 & 26 & 42 & 5 & 1 \\
\hline & $8-10$ & 17.5 & 12.5 & 4 & fat clay & 53 & 21 & 32 & 40 & 8 & 1 \\
\hline & 10-13 & 17.11 & 11.9 & 8 & fat clay & 56 & 28 & 28 & 43 & 6 & 1 \\
\hline & $16-20$ & 16.8 & 12.5 & 14 & fat clay & 57 & 26 & 31 & 36 & 6 & 1 \\
\hline & $20-23$ & 16.8 & 14.6 & 6 & fat clay & 44 & 21 & 23 & 47 & 5 & 1 \\
\hline & $23-26$ & 18.4 & 14.5 & 17 & fat clay & 34 & 19 & 15 & 27 & 8 & 1 \\
\hline & $26-30$ & 18.6 & 15.3 & 60 & silty sand & - & - & - & 21 & 1 & 16 \\
\hline & $30-40$ & 19 & 16 & 65 & silty sand & - & - & - & 18 & 1.5 & 30 \\
\hline
\end{tabular}




\section{Geotechnical Characteristics of Flat Plain Deposit}

Flat plain Deposits were deposited in recent time and form blankets in the delta area. They occupy the areas which are caused by tidal action. It is estimated to be about thirty-metrethick with variation according to depositional environments. This formation consists essentially of yellowish grey silt and clay. It also contains organic matter. Traces of sand are found scattered throughout the deposits. Table 7 and 8 show the summary of geotechnical characteristics of flat plain deposit in Yangon area.

Table 7. Summary of Geotechnical Characteristics of Flat Plain Deposit, Yakin Township in Yangon Area

\begin{tabular}{|c|c|c|c|c|c|c|c|c|c|c|c|}
\hline \multirow{2}{*}{ No. } & \multirow{2}{*}{$\begin{array}{c}\text { Depth } \\
\text { (m) }\end{array}$} & \multirow{2}{*}{$\begin{array}{c}\text { Wet } \\
\text { Unit } \\
\text { Weight } \\
\text { kN/m } / \mathbf{m}^{3}\end{array}$} & \multirow{2}{*}{$\begin{array}{c}\text { Dry } \\
\text { Unit } \\
\text { Weight } \\
\text { kN/m }\end{array}$} & \multirow{2}{*}{$\begin{array}{c}\text { SPT } \\
\mathbf{N} \\
\text { Value }\end{array}$} & \multirow{2}{*}{ Lithology } & \multicolumn{3}{|c|}{$\begin{array}{l}\text { Atterberg } \\
\text { Limits }\end{array}$} & \multirow{2}{*}{$\begin{array}{c}\text { Moisture } \\
\text { Content } \\
(\%)\end{array}$} & \multicolumn{2}{|c|}{$\begin{array}{c}\text { Shear } \\
\text { Strength }\end{array}$} \\
\hline & & & & & & $\begin{array}{c}\text { L.L } \\
\%\end{array}$ & $\begin{array}{c}\text { P.L } \\
\%\end{array}$ & P.I & & $\begin{array}{c}\mathrm{c} \\
\mathrm{kPa}\end{array}$ & $\begin{array}{c}\phi \\
\left(^{\circ}\right)\end{array}$ \\
\hline \multirow{5}{*}{ BH-1 } & $0-2$ & 17.6 & 14 & $10-15$ & sandy silt & - & - & - & $24-28$ & - & - \\
\hline & $2-4$ & 17.9 & 14.6 & $16-17$ & clayey silt & 43 & 23 & 20 & $22-23$ & 1.1 & 10 \\
\hline & $4-6$ & 18 & 14.8 & $18-21$ & clayey silt & 42 & 22 & 20 & $20-23$ & 1.0 & 20 \\
\hline & $6-8$ & 19 & 15.2 & $18-19$ & clayey silt & 40 & 23 & 17 & $20-22$ & 1.3 & 15 \\
\hline & $8-10$ & 19.2 & 15.4 & 20 & clayey silt & 40 & 23 & 17 & 20 & 1.4 & 18 \\
\hline \multirow[t]{5}{*}{ BH-2 } & $0-2$ & 16.5 & 13.4 & $3-13$ & $\begin{array}{l}\text { Clayey } \\
\text { sand }\end{array}$ & - & - & - & $25-33$ & - & - \\
\hline & $2-4$ & 17.8 & 14.5 & $15-16$ & $\begin{array}{l}\text { sandy and } \\
\text { clayey silt }\end{array}$ & 43 & 23 & 20 & $22-24$ & 1.0 & 18 \\
\hline & $4-6$ & 17.9 & 14.6 & $16-17$ & clayey silt & 44 & 24 & 20 & $21-24$ & 1.0 & 18 \\
\hline & $6-8$ & 18 & 15 & 17 & clayey silt & 45 & 24 & 21 & $20-21$ & 1.2 & 9 \\
\hline & $8-10$ & 18.3 & 15.6 & 18 & clayey silt & 45 & 24 & 21 & 21 & 1.2 & 12 \\
\hline
\end{tabular}

Table 8. Summary of Geotechnical Characteristics of Flat Plain Deposits, North Okkalapa, in Yangon Area

\begin{tabular}{|c|c|c|c|c|c|c|c|c|c|c|c|}
\hline \multirow[b]{2}{*}{ No. } & \multirow[b]{2}{*}{$\begin{array}{l}\text { Depth } \\
\text { (m) }\end{array}$} & \multirow{2}{*}{$\begin{array}{c}\text { Wet } \\
\text { Unit } \\
\text { Weight } \\
\text { kN/m }\end{array}$} & \multirow{2}{*}{$\begin{array}{c}\text { Dry } \\
\text { Unit } \\
\text { Weight } \\
\text { kN/m } / \mathbf{m}^{3}\end{array}$} & \multirow{2}{*}{$\begin{array}{c}\text { SPT } \\
\mathbf{N} \\
\text { Value }\end{array}$} & \multirow[b]{2}{*}{ Lithology } & \multicolumn{3}{|c|}{$\begin{array}{c}\text { Atterberg } \\
\text { Limits }\end{array}$} & \multirow{2}{*}{$\begin{array}{c}\text { Moisture } \\
\text { Content } \\
(\%)\end{array}$} & \multicolumn{2}{|c|}{$\begin{array}{c}\text { Shear } \\
\text { Strength }\end{array}$} \\
\hline & & & & & & $\begin{array}{l}\text { L.L } \\
\%\end{array}$ & $\begin{array}{l}\text { P.L } \\
\%\end{array}$ & P.I & & $\begin{array}{c}\mathrm{c} \\
\mathrm{kPa}\end{array}$ & $\begin{array}{l}\phi \\
\left({ }^{\circ}\right)\end{array}$ \\
\hline \multirow{5}{*}{ BH-1 } & $0-2$ & 16.3 & 12.9 & $9-10$ & clayey silt & 45 & 24 & 21 & $23-26$ & 1.0 & 10 \\
\hline & $2-4$ & 18.2 & 14.8 & 10 & clayey silt & 46 & 25 & 21 & $22-24$ & 1.2 & 11 \\
\hline & $4-6$ & 18.5 & 15 & 10 & $\begin{array}{l}\text { Sandy and } \\
\text { clayey silt }\end{array}$ & 48 & 25 & 23 & $22-23$ & 1.3 & 13 \\
\hline & $6-8$ & 18.6 & 15.4 & 10 & $\begin{array}{l}\text { Sandy and } \\
\text { clayey silt }\end{array}$ & 49 & 25 & 24 & $20-22$ & 1.5 & 8 \\
\hline & $8-10$ & 19 & 15.7 & 12 & clayey silt & 49 & 25 & 24 & 22 & 1.5 & 10 \\
\hline \multirow[t]{5}{*}{ BH-2 } & $0-2$ & 16.3 & 13 & 9 & $\begin{array}{l}\text { Silty sand } \\
\text { with clay }\end{array}$ & 44 & 24 & 20 & $25-26$ & 1.2 & 11 \\
\hline & $2-4$ & 18.2 & 14.8 & $9-10$ & Clayey silt & 46 & 25 & 21 & $23-25$ & 1.3 & 13 \\
\hline & $4-6$ & 18.5 & 15.1 & $10-11$ & Clayey silt & 48 & 25 & 23 & $23-24$ & 1.5 & 8 \\
\hline & $6-8$ & 18.6 & 15.2 & $11-12$ & Clayey silt & 49 & 25 & 24 & $22-23$ & 1.7 & 11 \\
\hline & $8-10$ & 19.2 & 16 & 13 & Clayey silt & 49 & 25 & 24 & 23 & 2.0 & 13 \\
\hline
\end{tabular}




\section{Analysis of Geotechnical Properties of Alluvial Deposits}

Bearing capacity of soil is defined as a unit load which can be produced safely without detrimental deformation to the structure on the surface of soil [6]. Bearing capacity may also be defined as the capacity of soil to withstand building without undergoing excessive settlement or shear failure. It is the most important field property that needs firm evaluation before any construction program is proposed over a soil. Geotechnical investigations are performed to obtain data on physical characteristics and mechanical properties of soil around a site to evaluate the bearing capacity of soil for the foundation analysis. From the borehole data, the recorded SPT values are corrected by using the following equation to obtain the exact information for foundation analysis.

$$
N_{60}=1.67 E_{m} C_{b} C_{r} N
$$

Where,

$$
\begin{aligned}
\mathrm{E}_{\mathrm{m}}= & \text { correction for hammer efficiency }(0.6 \text { for a safety hammer, } 0.45 \text { for donut } \\
& \text { hammer }) \\
\mathrm{C}_{\mathrm{b}}= & \text { correction for borehole diameter }(1.0 \text { for } 65 \mathrm{~mm}<\mathrm{D}<115 \mathrm{~mm}, 1.05 \text { for } \mathrm{D}= \\
& 150 \mathrm{~mm}, 1.15 \text { for } \mathrm{D}=200 \mathrm{~mm}) \\
\mathrm{C}_{\mathrm{r}}= & \text { correction for drill rod length }(1.0 \text { for } \mathrm{L}>10 \mathrm{~m}, 0.95 \text { for } 6 \mathrm{~m}<\mathrm{L}<10 \mathrm{~m}, 0.85 \\
& \text { for } 4 \mathrm{~m}<\mathrm{L}<6 \mathrm{~m}, 0.75 \text { for } \mathrm{L}<4 \mathrm{~m}) \\
\mathrm{N}= & \text { measured SPT blow count }
\end{aligned}
$$

The corrected value should be adjusted using an overburden correction that compensate for the effective stress. By using the following equation, the corrected $\mathrm{N}$ ( $\left.\mathbf{N}_{60}\right)$ value change into ( $\mathbf{N}_{1}$ ) 60 to calculate the bearing capacity of soil [ 7 ].

$$
\begin{aligned}
\left(\mathbf{N}_{1}\right) \mathbf{6 0}_{0}=\mathrm{C}_{\mathrm{n}} \times \mathbf{N}_{\mathbf{6 0}} \\
\text { where, } \begin{aligned}
\mathrm{C}_{\mathrm{n}} & =\sqrt{\frac{1}{\sigma^{\prime} v}} \\
\mathrm{C}_{\mathrm{n}} & =\text { overburden correction } \\
\delta^{\prime} v & =\text { effective overburden pressure }
\end{aligned}
\end{aligned}
$$

The ultimate bearing capacity is calculated by using the equation:

$$
\mathrm{Qult}_{\text {ult }}=\left(\mathrm{N}_{1}\right) 60 \times 12 \mathrm{kPa}
$$

\section{Geotechnical Properties of Valley Fill Deposits}

Table $9,10,11$, and 12 show the ultimate bearing capacity of valley fill deposits.

Table 9. Ultimate Bearing Capacity of Valley Fill Deposits in Kamaryut Township

\begin{tabular}{|ccccccccccccc|}
\hline No. & Depth $_{(\mathbf{m})}$ & $\mathbf{E}_{\mathbf{m}}$ & $\mathbf{C b}$ & $\mathbf{C r}$ & $\mathbf{N}$ & $\mathbf{N}_{\mathbf{6 0}}$ & $\boldsymbol{\gamma}\left(\mathbf{k N} / \mathbf{m}^{\mathbf{3}}\right)$ & $\boldsymbol{\delta} \boldsymbol{v}$ & $\boldsymbol{\delta}^{\prime} \boldsymbol{v}$ & $\mathbf{C}_{\mathbf{N}}$ & $\left(\mathbf{N}_{\mathbf{1}}\right)_{\mathbf{6 0}}$ & $\mathbf{Q}_{\text {ultt(kPa) }}$ \\
\hline BH-1 & 0 & 0.45 & 1 & 0.75 & 0 & 0 & 0 & 0 & 0 & 0 & 0 & 0 \\
& 1 & 0.45 & 1 & 0.75 & 8 & 4.5 & 16.8 & 16.8 & 16.8 & 2 & 9.018 & 108.22 \\
& 2 & 0.45 & 1 & 0.75 & 11 & 6.2 & 17.4 & 34.8 & 34.8 & 1.66 & 10.28 & 123.34 \\
& 3 & 0.45 & 1 & 0.75 & 15 & 8.5 & 17.8 & 7.8 & 42.6 & 1.5 & 12.67 & 152.02 \\
& 4 & 0.45 & 1 & 0.85 & 16 & 10 & 18 & 16 & 50.8 & 1.37 & 14.02 & 168.29 \\
& 5 & 0.45 & 1 & 0.85 & 17 & 11 & 18.2 & 24.6 & 59.4 & 1.27 & 13.78 & 165.36 \\
& 6 & 0.45 & 1 & 0.95 & 18 & 13 & 18.6 & 34.4 & 69.2 & 1.18 & 15.11 & 181.3 \\
& 7 & 0.45 & 1 & 0.95 & 19 & 14 & 19.1 & 45.5 & 80.3 & 1.09 & 14.8 & 177.65 \\
& 10 & 0.45 & 1 & 0.95 & 21 & 15 & 19.4 & 75.2 & 110 & 0.93 & 13.98 & 167.76 \\
\hline
\end{tabular}


Table 9. (Continued)

\begin{tabular}{|ccccccccccccc|}
\hline No. & Depth $_{(\mathbf{m})}$ & $\mathbf{E}_{\mathbf{m}}$ & $\mathbf{C b}$ & $\mathbf{C r}$ & $\mathbf{N}$ & $\mathbf{N}_{\mathbf{6 0}}$ & $\boldsymbol{\gamma}\left(\mathbf{k N} / \mathbf{m}^{\mathbf{3}}\right)$ & $\boldsymbol{\delta} \boldsymbol{v}$ & $\boldsymbol{\delta}^{\prime} \boldsymbol{v}$ & $\mathbf{C}_{\mathbf{N}}$ & $\left.\mathbf{( N}_{\mathbf{1}}\right)_{\mathbf{6}}$ & $\left.\mathbf{Q}_{\text {ult }} \mathbf{k P a}\right)$ \\
\hline BH-2 & 0 & 0.45 & 1 & 0.75 & 0 & 0 & 0 & 0 & 0 & 0 & 0 & 0 \\
& 1 & 0.45 & 1 & 0.75 & 11 & 6.2 & 17.3 & 17.1 & 17.1 & 2 & 12.4 & 148.8 \\
& 2 & 0.45 & 1 & 0.75 & 14 & 7.9 & 17.4 & 35.8 & 35.8 & 1.63 & 12.9 & 154.77 \\
& 3 & 0.45 & 1 & 0.75 & 15 & 8.5 & 17.6 & 52.8 & 52.8 & 1.35 & 11.38 & 136.55 \\
& 4 & 0.45 & 1 & 0.85 & 16 & 10 & 18 & 72 & 72 & 1.15 & 11.78 & 141.36 \\
& 5 & 0.45 & 1 & 0.85 & 17 & 11 & 18.2 & 91 & 91 & 1.03 & 11.13 & 133.6 \\
& 6 & 0.45 & 1 & 0.95 & 18 & 13 & 18.8 & 8.8 & 99.8 & 0.98 & 12.58 & 150.97 \\
& 7 & 0.45 & 1 & 0.95 & 19 & 14 & 19.1 & 18.2 & 109.2 & 0.94 & 12.7 & 152.34 \\
& 10 & 0.45 & 1 & 0.95 & 17 & 12 & 19.3 & 46.5 & 137.5 & 0.83 & 10.12 & 121.47 \\
\hline
\end{tabular}

$\mathrm{C}_{\mathrm{n}} \quad=$ overburden correction

$\delta^{\prime} v=$ effective overburden pressure

$\delta v=$ overburden pressure

Table 10. Ultimate Bearing Capacity of Valley Fill Deposits in Insein Township

\begin{tabular}{|c|c|c|c|c|c|c|c|c|c|c|c|c|}
\hline No. & $\operatorname{Depth}_{(\mathrm{m})}$ & $\mathbf{E}_{\mathbf{m}}$ & $\mathbf{C b}$ & $\mathrm{Cr}$ & $\mathbf{N}$ & $N_{60}$ & $\gamma\left(\mathbf{k N} / \mathbf{m}^{3}\right)$ & $\delta v$ & $\delta^{\prime} v$ & $\mathbf{C}_{\mathrm{N}}$ & $\left(\mathrm{N}_{1}\right)_{60}$ & Qult(kPa) \\
\hline \multirow{11}{*}{ BH-1 } & 0 & 0.45 & 1 & 0.75 & 0 & 0 & 0 & 0 & 0 & 0 & 0 & 0 \\
\hline & 1 & 0.45 & 1 & 0.75 & 8 & 4.5 & 17.4 & 17.4 & 17.4 & 2 & 9.018 & 108.2 \\
\hline & 2 & 0.45 & 1 & 0.75 & 13 & 7.3 & 17.6 & 7.6 & 25 & 1.96 & 14.33 & 172 \\
\hline & 3 & 0.45 & 1 & 0.75 & 16 & 9 & 17.9 & 15.8 & 33.2 & 1.7 & 15.31 & 183.7 \\
\hline & 4 & 0.45 & 1 & 0.75 & 12 & 6.8 & 17.4 & 22.2 & 39.6 & 1.55 & 10.51 & 126.1 \\
\hline & 5 & 0.45 & 1 & 0.85 & 9 & 5.7 & 17.4 & 29.6 & 47 & 1.43 & 8.201 & 98.42 \\
\hline & 6 & 0.45 & 1 & 0.85 & 16 & 10 & 17 & 35 & 52.4 & 1.35 & 13.81 & 165.7 \\
\hline & 7 & 0.45 & 1 & 0.85 & 16 & 10 & 17.9 & 47.4 & 64.8 & 1.21 & 12.42 & 149 \\
\hline & 8 & 0.45 & 1 & 0.85 & 16 & 10 & 17.9 & 55.3 & 72.7 & 1.15 & 11.72 & 140.7 \\
\hline & 9 & 0.45 & 1 & 0.95 & 16 & 11 & 17.9 & 63.2 & 80.6 & 1.09 & 12.44 & 149.3 \\
\hline & 10 & 0.45 & 1 & 0.95 & 16 & 11 & 17.9 & 71.1 & 88.5 & 1.04 & 11.88 & 142.5 \\
\hline \multirow{11}{*}{ BH-2 } & 0 & 0.45 & 1 & 0.75 & 0 & 0 & 0 & 0 & 0 & 0 & 0 & 0 \\
\hline & 1 & 0.45 & 1 & 0.75 & 11 & 6.2 & 17.1 & 17.1 & 17.1 & 0.24 & 1.499 & 17.99 \\
\hline & 2 & 0.45 & 1 & 0.75 & 14 & 7.9 & 17.5 & 5.25 & 22.4 & 0.21 & 1.669 & 20.03 \\
\hline & 3 & 0.45 & 1 & 0.75 & 15 & 8.5 & 17.5 & 12.8 & 29.9 & 0.18 & 1.547 & 18.57 \\
\hline & 4 & 0.45 & 1 & 0.75 & 16 & 9 & 18 & 21.6 & 38.7 & 0.16 & 1.45 & 17.4 \\
\hline & 5 & 0.45 & 1 & 0.85 & 17 & 11 & 18.1 & 30 & 47.1 & 0.15 & 1.583 & 18.99 \\
\hline & 6 & 0.45 & 1 & 0.85 & 18 & 11 & 18.1 & 38.1 & 55.2 & 0.13 & 1.548 & 18.58 \\
\hline & 7 & 0.45 & 1 & 0.85 & 19 & 12 & 18.8 & 50.2 & 67.3 & 0.12 & 1.48 & 17.76 \\
\hline & 8 & 0.45 & 1 & 0.85 & 18 & 11 & 19.3 & 62.3 & 79.4 & 0.11 & 1.29 & 15.48 \\
\hline & 9 & 0.45 & 1 & 0.95 & 17 & 12 & 18.6 & 66.2 & 83.3 & 0.11 & 1.33 & 15.96 \\
\hline & 10 & 0.45 & 1 & 0.95 & 17 & 12 & 18.6 & 74.8 & 91.9 & 0.1 & 1.266 & 15.19 \\
\hline
\end{tabular}

Table 11. Ultimate Bearing Capacity of Valley Fill Deposits in Ahlon Township

\begin{tabular}{|c|c|c|c|c|c|c|c|c|c|c|c|c|}
\hline No. & $\operatorname{Depth}_{(m)}$ & $\mathbf{E}_{\mathbf{m}}$ & $\mathrm{Cb}$ & $\mathrm{Cr}$ & $\mathbf{N}$ & $\mathbf{N}_{60}$ & $\gamma\left(\mathbf{k N} / \mathbf{m}^{3}\right)$ & $\delta v$ & $\delta^{\prime} v$ & $\mathrm{C}_{\mathrm{N}}$ & $\left(\mathrm{N}_{1}\right)_{60}$ & $Q_{\text {ult(kPa) }}$ \\
\hline \multirow{10}{*}{ BH-1 } & 1 & 0.45 & 1 & 0.75 & 5 & 2.82 & 16.98 & 16.98 & 16.98 & 2 & 5.6 & 67.63 \\
\hline & 2 & 0.45 & 1 & 0.75 & 4 & 2.25 & 16.83 & 6.83 & 23.81 & 2 & 4.5 & 54.22 \\
\hline & 3 & 0.45 & 1 & 0.75 & 4 & 2.25 & 16.77 & 13.54 & 30.52 & 1.77 & 4.0 & 47.89 \\
\hline & 4 & 0.45 & 1 & 0.85 & 6 & 3.83 & 16.64 & 19.92 & 36.9 & 1.61 & 6.2 & 74.05 \\
\hline & 5 & 0.45 & 1 & 0.85 & 16 & 10.2 & 17.9 & 31.6 & 48.58 & 1.4 & 14.3 & 172.09 \\
\hline & 6 & 0.45 & 1 & 0.95 & 17 & 12.1 & 16 & 40.55 & 57.53 & 1.29 & 15 & 187.79 \\
\hline & 7 & 0.45 & 1 & 0.95 & 18 & 12.9 & 18.22 & 49.32 & 66.3 & 1.2 & 15.4 & 185.22 \\
\hline & 8 & 0.45 & 1 & 0.95 & 19 & 13.6 & 18.58 & 60.06 & 77.04 & 1.11 & 15.1 & 181.37 \\
\hline & 9 & 0.45 & 1 & 0.95 & 18 & 12.9 & 19.05 & 72.4 & 89.38 & 1.03 & 13.2 & 159.52 \\
\hline & 10 & 0.45 & 1 & 0.95 & 18 & 12.9 & 18.38 & 75.42 & 92.4 & 1.02 & 13.0 & 156.9 \\
\hline
\end{tabular}


Table 11. (Continued)

\begin{tabular}{|ccccccccccccc|}
\hline No. & Depth $_{(\mathbf{m})}$ & $\mathbf{E}_{\mathbf{m}}$ & $\mathbf{C b}$ & $\mathbf{C r}$ & $\mathbf{N}$ & $\mathbf{N}_{\mathbf{6 0}}$ & $\gamma\left(\mathbf{k N} / \mathbf{m}^{\mathbf{3}}\right)$ & $\delta \boldsymbol{v}$ & $\boldsymbol{\delta}^{\prime} \boldsymbol{v}$ & $\mathbf{C}_{\mathbf{N}}$ & $\left.\mathbf{( N}_{\mathbf{1}}\right)_{\mathbf{6 0}}$ & $\mathbf{Q}_{\mathbf{u l t}(\mathbf{k P a})}$ \\
\hline & 1 & 0.45 & 1 & 0.75 & 12 & 6.76 & 17.6 & 5.32 & 5.32 & 2 & 13.53 & 162.32 \\
$\mathrm{BH}-2$ & 2 & 0.45 & 1 & 0.75 & 13 & 7.33 & 17.71 & 13.11 & 18.43 & 2 & 14.65 & 175.85 \\
& 3 & 0.45 & 1 & 0.75 & 18 & 10.1 & 18.44 & 22.79 & 28.11 & 1.84 & 18.71 & 224.58 \\
& 4 & 0.45 & 1 & 0.85 & 18 & 11.5 & 18.54 & 31.6 & 36.92 & 1.61 & 18.51 & 222.09 \\
& 5 & 0.45 & 1 & 0.85 & 17 & 10.9 & 18.13 & 38.21 & 43.53 & 1.48 & 16.1 & 193.16 \\
& 6 & 0.45 & 1 & 0.95 & 18 & 12.9 & 18.4 & 47.88 & 53.2 & 1.34 & 17.23 & 206.77 \\
& 7 & 0.45 & 1 & 0.95 & 19 & 13.6 & 18.52 & 57.08 & 62.4 & 1.24 & 16.79 & 201.52 \\
& 8 & 0.45 & 1 & 0.95 & 19 & 13.6 & 18.54 & 65.76 & 71.08 & 1.16 & 15.74 & 188.82 \\
& 9 & 0.45 & 1 & 0.95 & 19 & 13.6 & 19.17 & 79.78 & 85.1 & 1.06 & 14.38 & 172.57 \\
& 10 & 0.45 & 1 & 0.95 & 20 & 14.3 & 19.35 & 90.7 & 96.02 & 1 & 14.25 & 171.01 \\
\hline
\end{tabular}

Table 12. Ultimate Bearing Capacity of Valley Filled Deposits in Kuauktada

Township

\begin{tabular}{|ccccccccccccc|}
\hline No. & Depth $_{(\mathbf{m})}$ & $\mathbf{E}_{\mathbf{m}}$ & $\mathbf{C}_{\mathbf{b}}$ & $\mathbf{C}_{\mathbf{r}}$ & $\mathbf{N}$ & $\mathbf{N}_{\mathbf{6 0}}$ & $\gamma\left(\mathbf{k N} / \mathbf{m}^{\mathbf{3}}\right)$ & $\boldsymbol{\delta} \boldsymbol{v}$ & $\boldsymbol{\delta}^{\prime} \mathbf{v}$ & $\mathbf{C}_{\mathbf{N}}$ & $\left.\mathbf{( N}_{\mathbf{1}}\right)_{\mathbf{6} \mathbf{0}}$ & $\mathbf{Q}_{\mathbf{u l t}(\mathbf{k P a})}$ \\
\hline & 0 & 0.45 & 1 & 0.75 & 0 & 0 & 0 & 0 & 0 & 0 & 0 & 0 \\
BH-1 & 1 & 0.45 & 1 & 0.75 & 4 & 2.25 & 17.25 & 16.98 & 17 & 2 & 4.51 & 54.108 \\
& 2 & 0.45 & 1 & 0.75 & 6 & 3.38 & 17.69 & 5.383 & 22.4 & 2 & 6.76 & 81.162 \\
& 3 & 0.45 & 1 & 0.75 & 8 & 4.51 & 17.75 & 13.18 & 30.2 & 1.78 & 8.03 & 96.365 \\
& 4 & 0.45 & 1 & 0.85 & 13 & 8.3 & 17.89 & 21.3 & 38.3 & 1.58 & 13.1 & 157.51 \\
& 5 & 0.45 & 1 & 0.85 & 16 & 10.2 & 18.13 & 30.08 & 47.1 & 1.43 & 14.6 & 174.85 \\
& 6 & 0.45 & 1 & 0.95 & 16 & 11.4 & 18.3 & 39.01 & 56 & 1.31 & 14.9 & 179.16 \\
& 7 & 0.45 & 1 & 0.95 & 17 & 12.1 & 18.63 & 49.19 & 66.2 & 1.2 & 14.6 & 175.1 \\
& 8 & 0.45 & 1 & 0.95 & 20 & 14.3 & 19.04 & 60.57 & 77.5 & 1.11 & 15.9 & 190.29 \\
& 9 & 0.45 & 1 & 0.95 & 20 & 14.3 & 19.31 & 71.69 & 88.7 & 1.04 & 14.8 & 177.96 \\
& 10 & 0.45 & 1 & 1 & 21 & 15.8 & 19.37 & 81.52 & 98.5 & 0.99 & 15.6 & 186.62 \\
\hline & 1 & 0.45 & 1 & 0.75 & 10 & 5.64 & 17.44 & 2.232 & 5.32 & 2 & 11.3 & 135.27 \\
& 2 & 0.45 & 1 & 0.75 & 6 & 3.38 & 17.28 & 9.464 & 14.8 & 2 & 6.76 & 81.162 \\
& 3 & 0.45 & 1 & 0.75 & 12 & 6.76 & 17.58 & 17.43 & 22.8 & 2.05 & 13.9 & 166.4 \\
& 4 & 0.45 & 1 & 0.85 & 16 & 10.2 & 17.97 & 26.3 & 31.6 & 1.74 & 17.8 & 213.3 \\
& 5 & 0.45 & 1 & 0.85 & 16 & 10.2 & 17.98 & 34.31 & 39.6 & 1.55 & 15.9 & 190.53 \\
& 6 & 0.45 & 1 & 0.95 & 13 & 9.28 & 17.55 & 40.02 & 45.3 & 1.45 & 13.5 & 161.77 \\
& 7 & 0.45 & 1 & 0.95 & 12 & 8.57 & 17.56 & 47.63 & 52.9 & 1.34 & 11.5 & 138.17 \\
& 8 & 0.45 & 1 & 0.95 & 13 & 9.28 & 17.6 & 55.48 & 60.8 & 1.25 & 11.6 & 139.69 \\
& 9 & 0.45 & 1 & 0.95 & 14 & 9.99 & 17.74 & 64.24 & 69.6 & 1.17 & 11.7 & 140.64 \\
& 10 & 0.45 & 1 & 1 & 14 & 10.5 & 17.81 & 72.63 & 78 & 1.11 & 11.7 & 139.85 \\
\hline
\end{tabular}

\section{Geotechnical Properties of Flat Plain Deposits}

Table 13 and 14 show the ultimate bearing capacity of flat plain deposits.

Table 13. Ultimate Bearing Capacity of Flat Plain Deposits in Yakin Township

\begin{tabular}{|ccccccccccccc|}
\hline No. & Depth $_{(\mathbf{m})}$ & $\mathbf{E}_{\mathbf{m}}$ & $\mathbf{C}_{\mathbf{b}}$ & $\mathbf{C}_{\mathbf{r}}$ & $\mathbf{N}$ & $\mathbf{N}_{\mathbf{6 0}}$ & $\gamma\left(\mathbf{k N} / \mathbf{m}^{3}\right)$ & $\delta \boldsymbol{v}$ & $\delta^{\prime} \boldsymbol{v}$ & $\mathbf{C}_{\mathbf{N}}$ & $\left(\mathbf{N}_{\mathbf{1}}\right)_{\mathbf{6 0}}$ & $\mathbf{Q}_{\mathbf{u l t}(\mathbf{k P a})}$ \\
\hline \multirow{3}{*}{ BH-1 } & 0 & 0.45 & 1 & 0.75 & 0 & 0 & 0 & 0 & 0 & 0 & 0 & 0 \\
& 1 & 0.45 & 1 & 0.75 & 5 & 2.82 & 19.6 & 19.6 & 19.6 & 2 & 5.64 & 67.635 \\
& 2 & 0.45 & 1 & 0.75 & 5 & 2.82 & 19.6 & 9.6 & 29.2 & 2 & 5.64 & 67.635 \\
& 3 & 0.45 & 1 & 0.75 & 9 & 5.07 & 19.6 & 19.2 & 38.8 & 1.57 & 7.96 & 95.573 \\
& 5 & 0.45 & 1 & 0.85 & 6 & 3.83 & 19.6 & 38.4 & 58 & 1.28 & 4.92 & 59.062 \\
& 7 & 0.45 & 1 & 0.95 & 5 & 3.57 & 19.6 & 57.6 & 77.2 & 1.11 & 3.97 & 47.68 \\
& 8 & 0.45 & 1 & 0.95 & 5 & 3.57 & 19.6 & 67.2 & 86.8 & 1.05 & 3.75 & 44.966 \\
& 9 & 0.45 & 1 & 0.95 & 6 & 4.28 & 19.6 & 76.8 & 96.4 & 1 & 4.27 & 51.202 \\
& 10 & 0.45 & 1 & 0.95 & 6 & 4.28 & 19.65 & 86.9 & 106 & 0.95 & 4.06 & 48.725 \\
\hline
\end{tabular}


Table 13. (Continued)

\begin{tabular}{|ccccccccccccc|}
\hline No. & Depth $_{(\mathbf{m})}$ & $\mathbf{E}_{\mathbf{m}}$ & $\mathbf{C}_{\mathbf{b}}$ & $\mathbf{C}_{\mathbf{r}}$ & $\mathbf{N}$ & $\mathbf{N}_{\mathbf{6 0}}$ & $\gamma\left(\mathbf{k N} / \mathbf{m}^{3}\right)$ & $\delta \boldsymbol{v}$ & $\boldsymbol{\delta}^{\prime} \boldsymbol{v}$ & $\mathbf{C}_{\mathbf{N}}$ & $\mathbf{( N}_{\mathbf{1}} \mathbf{6}_{\mathbf{6}}$ & $\mathbf{Q}_{\mathbf{u l t}(\mathbf{k P a})}$ \\
\hline BH-2 & 0 & 0.45 & 1 & 0.75 & 0 & 0 & 0 & 0 & & 0 & 0 & 0 \\
& 1 & 0.45 & 1 & 0.75 & 3 & 1.69 & 19.7 & 19.7 & 19.7 & 2 & 3.38 & 40.581 \\
& 2 & 0.45 & 1 & 0.75 & 3 & 1.69 & 19.4 & 9.4 & 29.1 & 1.81 & 3.07 & 36.786 \\
& 3 & 0.45 & 1 & 0.75 & 5 & 2.82 & 19.4 & 18.8 & 38.5 & 1.58 & 4.44 & 53.303 \\
& 4 & 0.45 & 1 & 0.85 & 6 & 3.83 & 19.4 & 28.2 & 47.9 & 1.41 & 5.42 & 64.991 \\
& 5 & 0.45 & 1 & 0.85 & 7 & 4.47 & 19.4 & 37.6 & 57.3 & 1.29 & 5.78 & 69.325 \\
& 6 & 0.45 & 1 & 0.95 & 10 & 7.14 & 19.4 & 47 & 66.7 & 1.2 & 8.55 & 102.59 \\
& 7 & 0.45 & 1 & 0.95 & 14 & 9.99 & 19.4 & 56.4 & 76.1 & 1.12 & 11.2 & 134.46 \\
& 8 & 0.45 & 1 & 0.95 & 18 & 12.9 & 19.4 & 65.8 & 85.5 & 1.06 & 13.6 & 163.1 \\
& 9 & 0.45 & 1 & 0.95 & 24 & 17.1 & 19.4 & 75.2 & 94.9 & 1 & 17.2 & 206.42 \\
& 10 & 0.45 & 1 & 0.95 & 28 & 20 & 20.4 & 93.6 & 113 & 0.92 & 18.4 & 220.4 \\
\hline
\end{tabular}

Table 14. Ultimate Bearing Capacity of Flat Plain Deposits in N.Okkalarpa Township

\begin{tabular}{|ccccccccccccc|}
\hline No. & Depth $_{(\mathbf{m})}$ & $\mathbf{E}_{\mathbf{m}}$ & $\mathbf{C b}$ & $\mathbf{C r}$ & $\mathbf{N}$ & $\mathbf{N}_{\mathbf{6 0}}$ & $\gamma\left(\mathbf{k N} / \mathbf{m}^{\mathbf{3}}\right)$ & $\boldsymbol{\delta} \boldsymbol{v}$ & $\boldsymbol{\delta}^{\prime} \mathbf{v}$ & $\mathbf{C}_{\mathbf{N}}$ & $\mathbf{( N}_{\mathbf{1}} \mathbf{6}_{\mathbf{6}}$ & $\mathbf{Q}_{\mathbf{u l t}(\mathbf{k P a})}$ \\
\hline BH-1 & 0 & 0.45 & 1 & 0.75 & 0 & 0 & 0 & 0 & 0 & 0 & 0 & 0 \\
& 1 & 0.45 & 1 & 0.75 & 8 & 4.51 & 17.4 & 17.4 & 17.4 & 2 & 9.018 & 108.22 \\
& 2 & 0.45 & 1 & 0.75 & 13 & 7.33 & 17.6 & 7.6 & 25 & 2 & 14.33 & 171.98 \\
& 3 & 0.45 & 1 & 0.75 & 16 & 9.02 & 17.9 & 15.8 & 33.2 & 1.7 & 15.31 & 183.68 \\
& 4 & 0.45 & 1 & 0.75 & 12 & 6.76 & 17.4 & 22.2 & 39.6 & 1.6 & 10.51 & 126.14 \\
& 5 & 0.45 & 1 & 0.85 & 9 & 5.75 & 17.4 & 29.6 & 47 & 1.4 & 8.201 & 98.415 \\
& 6 & 0.45 & 1 & 0.85 & 16 & 10.2 & 17 & 35 & 52.4 & 1.4 & 13.81 & 165.7 \\
& 7 & 0.45 & 1 & 0.85 & 16 & 10.2 & 17.9 & 47.4 & 64.8 & 1.2 & 12.42 & 149 \\
& 8 & 0.45 & 1 & 0.85 & 16 & 10.2 & 17.9 & 55.3 & 72.7 & 1.1 & 11.72 & 140.68 \\
& 9 & 0.45 & 1 & 0.95 & 16 & 11.4 & 17.9 & 63.2 & 80.6 & 1.1 & 12.44 & 149.32 \\
& 10 & 0.45 & 1 & 0.95 & 16 & 11.4 & 17.9 & 71.1 & 88.5 & 1 & 11.88 & 142.5 \\
\hline & & & & & & & & & & & & \\
BH-2 & 0 & 0.45 & 1 & 0.75 & 0 & 0 & 0 & 0 & 0 & 0 & 0 & 0 \\
& 1 & 0.45 & 1 & 0.75 & 11 & 6.2 & 17.1 & 17.1 & 17.1 & 0.2 & 1.499 & 17.991 \\
& 2 & 0.45 & 1 & 0.75 & 14 & 7.89 & 17.5 & 5.25 & 22.4 & 0.2 & 1.669 & 20.029 \\
& 3 & 0.45 & 1 & 0.75 & 15 & 8.45 & 17.5 & 12.8 & 29.9 & 0.2 & 1.547 & 18.569 \\
& 4 & 0.45 & 1 & 0.75 & 16 & 9.02 & 18 & 21.6 & 38.7 & 0.2 & 1.45 & 17.395 \\
& 5 & 0.45 & 1 & 0.85 & 17 & 10.9 & 18.1 & 30 & 47.1 & 0.1 & 1.583 & 18.994 \\
& 6 & 0.45 & 1 & 0.85 & 18 & 11.5 & 18.1 & 38.1 & 55.2 & 0.1 & 1.548 & 18.576 \\
& 7 & 0.45 & 1 & 0.85 & 19 & 12.1 & 18.8 & 50.2 & 67.3 & 0.1 & 1.48 & 17.758 \\
& 8 & 0.45 & 1 & 0.85 & 18 & 11.5 & 19.3 & 62.3 & 79.4 & 0.1 & 1.29 & 15.483 \\
& 9 & 0.45 & 1 & 0.95 & 17 & 12.1 & 18.6 & 66.2 & 83.3 & 0.1 & 1.33 & 15.955 \\
\hline
\end{tabular}

\section{Geotechnical Properties of Marine Alluvial Deposits}

Table 15 and 16 show the ultimate bearing capacity of marine alluvial deposits.

Table 15. Ultimate Bearing Capacity of Marine Alluvium in Hlaingtharyar Township

\begin{tabular}{|ccccccccccccc|}
\hline No. & Depth $_{(\mathbf{m})}$ & $\mathbf{E}_{\mathbf{m}}$ & $\mathbf{C}_{\mathbf{b}}$ & $\mathbf{C}_{\mathbf{r}}$ & $\mathbf{N}$ & $\mathbf{N}_{\mathbf{6 0}}$ & $\boldsymbol{\gamma ( \mathbf { k N } / \mathbf { m } ^ { 3 } )}$ & $\boldsymbol{\delta} \boldsymbol{v}$ & $\boldsymbol{\delta}^{\prime} \boldsymbol{v}$ & $\mathbf{C}_{\mathbf{N}}$ & $\left(\mathbf{N}_{\mathbf{1}}\right)_{\mathbf{6 0}}$ & $\mathbf{Q}_{\mathbf{u l t}(\mathbf{k P a})}$ \\
\hline BH-1 & 0 & 0.45 & 1 & 0.75 & 0 & 0 & 0 & 0 & 0 & 0 & 0 & 0 \\
& 1 & 0.45 & 1 & 0.75 & 1 & 0.56 & 17.9 & 5.53 & 5.53 & 2 & 1.13 & 13.53 \\
& 2 & 0.45 & 1 & 0.75 & 2 & 1.13 & 17.9 & 13.43 & 18.96 & 2 & 2.25 & 27.05 \\
& 4 & 0.45 & 1 & 0.75 & 4 & 2.25 & 17.9 & 29.23 & 34.76 & 2 & 4.51 & 54.11 \\
& 6 & 0.45 & 1 & 0.85 & 2 & 1.28 & 17.2 & 41.04 & 46.57 & 1.4 & 1.83 & 21.97 \\
& 8 & 0.45 & 1 & 0.85 & 4 & 2.56 & 17.2 & 55.44 & 60.97 & 1.3 & 3.2 & 38.4 \\
& 10 & 0.45 & 1 & 0.95 & 3 & 2.14 & 17.6 & 73.72 & 79.25 & 1.1 & 2.35 & 28.24 \\
& 20 & 0.45 & 1 & 1 & 13 & 9.77 & 16.8 & 134 & 139.5 & 0.8 & 8.09 & 97.08 \\
& 30 & 0.45 & 1 & 1 & 42 & 31.6 & 19.36 & 278 & 283.5 & 0.6 & 18.3 & 220 \\
\hline
\end{tabular}


Table 15. (Continued)

\begin{tabular}{|ccccccccccccc|}
\hline No. & Depth $_{(\mathbf{m})}$ & $\mathbf{E}_{\mathbf{m}}$ & $\mathbf{C}_{\mathbf{b}}$ & $\mathbf{C}_{\mathbf{r}}$ & $\mathbf{N}$ & $\mathbf{N}_{\mathbf{6 0}}$ & $\gamma\left(\mathbf{k N} / \mathbf{m}^{3}\right)$ & $\delta \boldsymbol{v}$ & $\boldsymbol{\delta}^{\prime} \boldsymbol{v}$ & $\mathbf{C}_{\mathbf{N}}$ & $\left.\mathbf{( N}_{\mathbf{1}}\right)_{\mathbf{6} \mathbf{0}}$ & $\mathbf{Q}_{\mathbf{u l t}(\mathbf{k P a})}$ \\
\hline BH-2 & 0 & 0.45 & 1 & 0.75 & 0 & 0 & 0 & 0 & & 0 & 0 & 0 \\
& 1 & 0.45 & 1 & 0.75 & 0 & 0 & 18.14 & 18.14 & 18.14 & 2 & 0 & 0 \\
& 2 & 0.45 & 1 & 0.75 & 1 & 0.56 & 18.14 & 8.14 & 26.28 & 1.9 & 1.08 & 12.9 \\
& 4 & 0.45 & 1 & 0.75 & 3 & 1.69 & 18.1 & 24.3 & 42.44 & 1.5 & 2.54 & 30.46 \\
& 6 & 0.45 & 1 & 0.85 & 4 & 2.56 & 17.5 & 37.5 & 55.64 & 1.3 & 3.35 & 40.2 \\
& 8 & 0.45 & 1 & 0.85 & 3 & 1.92 & 17.2 & 50.4 & 68.54 & 1.2 & 2.26 & 27.17 \\
& 10 & 0.45 & 1 & 0.95 & 4 & 2.86 & 17.5 & 67.5 & 85.64 & 1.1 & 3.02 & 36.22 \\
& 20 & 0.45 & 1 & 1 & 14 & 10.5 & 16.8 & 129.2 & 147.3 & 0.8 & 8.48 & 101.7 \\
& 30 & 0.45 & 1 & 1 & 60 & 45.1 & 18.6 & 249.4 & 267.5 & 0.6 & 27 & 323.5 \\
\hline
\end{tabular}

Table 16. Ultimate Bearing Capacity of Marine Alluvial Deposits in Dagon Seikkan Township

\begin{tabular}{|c|c|c|c|c|c|c|c|c|c|c|c|c|}
\hline No. & $\operatorname{Depth}_{(\mathrm{m})}$ & $\mathbf{E}_{\mathbf{m}}$ & $\mathbf{C}_{\mathrm{b}}$ & $C_{r}$ & $\mathbf{N}$ & $\mathbf{N}_{60}$ & $\gamma\left(\kappa N / \mathbf{m}^{3}\right)$ & $\delta v$ & $\delta^{\prime} v$ & $\mathrm{C}_{\mathrm{N}}$ & $\left(\mathrm{N}_{1}\right)_{60}$ & $\mathbf{Q}_{\text {ult(kPa) }}$ \\
\hline \multirow{10}{*}{ BH-1 } & 2 & 0.45 & 1 & 0.75 & 5 & 2.82 & 17.9 & 7.9 & 25.8 & 1.9 & 5.43 & 65.113 \\
\hline & 3 & 0.45 & 1 & 0.75 & 5 & 2.82 & 17.9 & 16 & 33.7 & 1.7 & 4.75 & 56.973 \\
\hline & 4 & 0.45 & 1 & 0.85 & 5 & 3.19 & 17.9 & 23.7 & 41.6 & 1.5 & 4.84 & 58.115 \\
\hline & 5 & 0.45 & 1 & 0.85 & 3 & 1.92 & 17.8 & 31.2 & 49.1 & 1.4 & 2.67 & 32.096 \\
\hline & 6 & 0.45 & 1 & 0.95 & 3 & 2.14 & 17.4 & 37 & 54.9 & 1.3 & 2.83 & 33.924 \\
\hline & 7 & 0.45 & 1 & 0.95 & 5 & 3.57 & 17.4 & 44.4 & 62.3 & 1.2 & 4.42 & 53.076 \\
\hline & 10 & 0.45 & 1 & 0.95 & 5 & 3.57 & 18.8 & 79.2 & 97.1 & 1 & 3.54 & 42.514 \\
\hline & 30 & 0.45 & 1 & 1 & 20 & 15 & 18.1 & 235 & 252.8 & 0.6 & 9.25 & 110.94 \\
\hline & 40 & 0.45 & 1 & 1 & 26 & 19.5 & 18.5 & 332 & 349.4 & 0.5 & 10.2 & 122.68 \\
\hline & 80 & 0.45 & 1 & 1 & 59 & 44.3 & 20.3 & 814 & 831.6 & 0.3 & 15 & 180.44 \\
\hline \multirow{18}{*}{ BH-2 } & 0 & 0.45 & 1 & 0.75 & 0 & 0 & 0 & 0 & & 0 & 0 & 0 \\
\hline & 1 & 0.45 & 1 & 0.75 & 0 & 0 & 18.14 & 18.1 & 18.14 & 2 & 0 & 0 \\
\hline & 2 & 0.45 & 1 & 0.75 & 5 & 2.82 & 18.14 & 36.3 & 36.28 & 1.6 & 4.58 & 54.909 \\
\hline & 3 & 0.45 & 1 & 0.75 & 5 & 2.82 & 17 & 7 & 25.14 & 2 & 5.5 & 65.963 \\
\hline & 4 & 0.45 & 1 & 0.85 & 3 & 1.92 & 18 & 16 & 34.14 & 1.7 & 3.21 & 38.491 \\
\hline & 5 & 0.45 & 1 & 0.85 & 1 & 0.64 & 18 & 24 & 42.14 & 1.5 & 0.96 & 11.548 \\
\hline & 6 & 0.45 & 1 & 0.95 & 1 & 0.71 & 18.2 & 32.8 & 50.94 & 1.4 & 0.98 & 11.739 \\
\hline & 7 & 0.45 & 1 & 0.95 & 13 & 9.28 & 18.2 & 41 & 59.14 & 1.3 & 11.8 & 141.64 \\
\hline & 8 & 0.45 & 1 & 0.95 & 11 & 7.85 & 18.3 & 49.8 & 67.94 & 1.2 & 9.32 & 111.82 \\
\hline & 9 & 0.45 & 1 & 0.95 & 11 & 7.85 & 17.6 & 53.2 & 71.34 & 1.2 & 9.09 & 109.12 \\
\hline & 10 & 0.45 & 1 & 0.95 & 33 & 23.6 & 21 & 88 & 106.1 & 0.9 & 22.4 & 268.38 \\
\hline & 20 & 0.45 & 1 & 1 & 22 & 16.5 & 20.5 & 189 & 207.1 & 0.7 & 11.2 & 134.82 \\
\hline & 30 & 0.45 & 1 & 1 & 20 & 15 & 19 & 252 & 270.1 & 0.6 & 8.94 & 107.32 \\
\hline & 40 & 0.45 & 1 & 1 & 26 & 19.5 & 20.6 & 403 & 420.9 & 0.5 & 9.31 & 111.77 \\
\hline & 50 & 0.45 & 1 & 1 & 37 & 27.8 & 20.3 & 494 & 512.5 & 0.4 & 12 & 144.14 \\
\hline & 60 & 0.45 & 1 & 1 & 19 & 14.3 & 18.8 & 510 & 528.5 & 0.4 & 6.07 & 72.889 \\
\hline & 70 & 0.45 & 1 & 1 & 36 & 27.1 & 17.7 & 524 & 541.7 & 0.4 & 11.4 & 136.41 \\
\hline & 80 & 0.45 & 1 & 1 & 48 & 36.1 & 17.5 & 585 & 603.1 & 0.4 & 14.4 & 172.38 \\
\hline
\end{tabular}

\section{Conclusions and Recommendation}

Alluvium refers to loose soil or sediments that have been eroded and reshaped by moving water and moved to another. The running water carries the alluvial soil to a flood plan, where it spreads out and changes the flow of water around it. Therefore, in order to analyze the foundation of engineering structure, soil classification schemes in relation to mechanical properties of alluvial deposits are required to test before the construction. 
In Yangon area, the alluvial deposits are widely distributed. Mostly about two-third of Yangon area is covered by the alluvial deposits. The alluvial deposit in Yangon area is categorized into valley fill alluvial deposits, marine alluvial deposits, and flat plain alluvial deposits. The valley fill alluvium is found in Kamayut, Gyogon, Insein, Bahosi, Lammadaw, Hlaing, Ahlon, Kyauktada, and Botahtaung Townships. The marine alluvium is found in Simalaik, Bayintnaung, Shwepyithar, Hlaingtharyar, Hlagya, Htatabin and Dala Townships. The flat plain deposit is found in Yakin, North Okkalapa, South Okkalapa, Dagon Myothit, Thingangyun, Dawbon, and Phaunggyi Townships etc. The ultimate bearing capacity of valley fill deposits are more than $200 \mathrm{kPa}$ in Ahlon and Kyauktada Townships but less than $20 \mathrm{kPa}$ in some parts of Insein. The ultimate bearing capacity of flat plain deposits in Yakin is more than $200 \mathrm{kPa}$ but less than $20 \mathrm{kPa}$ in North Okkalarpa. Ultimate bearing capacity of some of marine alluvium in Hlaingtharyar and Dagonseikkan Townships are more than 200 $\mathrm{kPa}$ but some are less than $50 \mathrm{kPa}$. Most of bearing capacity of Hlaingtharyar, Insein and North Okkalarpa are less than $50 \mathrm{kPa}$. The most common soil types of alluvial deposits are CL, CH, SM, SC, ML according to the Unified Soil Classification. The geotechnical characteristics of alluvial deposits are carefully evaluated especially the Hlaingtharyar and North Okkalarpa Townships.

In some part of Hlaingtharyar and North Okkalarpa Townships, the groundwater level is high and the ultimate bearing capacity is less than $50 \mathrm{kPa}$. Since the most abundance of alluvial deposits in Yangon area, liquefaction may occur in some township. So, liquefaction potential analysis should be performed before construction of high rise building. According to the earthquake zonation map, Yangon is earthquake prone area. Therefore, earthquake resistant design should be evaluated. Moreover, systematic drainage system and systematic ground improvement methods should be designed.

\section{Acknowledgement}

The author's heartfelt thanks U Sann Lwin, Rtd Director, Head of Engineering Geology Office (1), Irrigation Department for their enthusiasm in sharing knowledge and expertise. I would like to extend special thanks to Dr Kyaw Htun, Rtd., Professor and Head, Department of Engineering Geology, Yangon Technological University for his guideline and cooperation. The author's deepest thanks are due to all persons for their excellent encouragements and supporting in carrying out this paper.

\section{References}

[1] D.F. Lapidus, Collins Dictionary of Geology, $2^{\text {nd }}$ Edition, Collins, Glasgow, United Kingdom, 2000.

[2] K.M. Bangar, Principle of Engineering Geology, $1^{\text {st }}$ Edition, Standard Publishers Distributors, New Delhi, India, 1995.

[3] Yangon City Development Committee, Yangon Townships Map, 1: 300000, Yangon, Myanmar: Yangon City Development Committee, 2015.

[4] W. Naing, Hydrogeology of Greater Yangon. Unpublished Master's Thesis, University of Yangon, Yangon, Myanmar, 1972.

[5] J.M. Trefethen, Geology for Engineers, $1^{\text {st }}$ Edition, Thomas Nelsons and Sons Ltd., London , 1972.

[6] I.S. Dunn, L.R. Anderson, and F.W. Kiefer, Fundamentals of Geotechnical Analysis, $1^{\text {st }}$ Edition, John Wiley \& Sons Inc., United States of America, 1980.

[7] V.N.S. Murthy, Geotechnical Engineering: Principle and Practices of Soil Mechanics and Foundation Engineering, $1^{\text {st }}$ Edition, Marcel Dekker Inc., New York, 2003. 